\section{Fracturas faciales por accidentes ciclísticos en pacientes pediátricos y adolescentes}

Facial fractures by bicycle accidents in pediatric and adolescent patients

\section{Resumen}

El objetivo de este estudio fue realizar un análisis retrospectivo de la incidencia, características, uso de dispositivos de seguridad, tratamiento y complicaciones de los traumatismos maxilofaciales causados por accidentes ciclísticos en una población pediátrica adolescente del Estado de São Paulo. La muestra fue seleccionada a partir del análisis de 2986 historias clínicas de pacientes víctimas de traumatismos faciales atendidos entre 1999 y 2008 por el Área de Círugia Buco Máxilo Facial de la Facultad de Odontología de Piracicaba, Universidad Estatal de Campinas, Estado de São Paulo-Brasil. Durante este periodo, 757 pacientes menores de 18 años fueron atendidos, de los cuales, 220 fueron víctimas de accidentes ciclísticos. Se conluyó que los accidentes ciclísticos representaron la principal etiología del trauma maxilofacial, siendo el género masculino predominante en todos los grupos etarios evaluados. Las fracturas mandibulares fueron las más prevalentes y la gran mayoría de pacientes no usaba ningún tipo de dispositivo de seguridad al momento del accidente.

Palabras Clave: Trauma facial, dispositivos de seguridad, nińos, fracturas de tercio medio, Brasil

\section{Abstract}

The aim of this study was a retrospective analysis of the incidence, characteristics, use of safety devices, treatment and complications of maxillofacial trauma caused by bicycle accidents in a pediatric and adolescent population of São Paulo. The sample was selected from analysis of 2986 medical records of victims of facial trauma patients treated entre 1999 and 2008 by the Department of Oral and Maxillofacial Surgery, Piracicaba Dental School, State University of Campinas, São Paulo, Brazil. During this period, 757 patients under 18 years were treated, of which 220 were victims of bicycle accidents. The bicycle accidents constituted the main etiology (29\%) of facial trauma. Only $1.8 \%$ of patients made use of safety devices. The adolescents group and male gender were the most prevalent in all age groups evaluated. Were identified 69 facial fractures, being jaw the most affected $(55.1 \%)$.

Conservative treatment was used in the most of cases (50 patients $72.4 \%$ ), while only one case of postoperative complication was reported.

Key words : Facial fracture, safety devices, children, middle third fractures, Brazil.
Artículo Original

\section{José Muñante-Cárdenas, ${ }^{1}$ Evandro Portella Figueirêdo, ${ }^{1}$ Sergio Olate, ${ }^{2}$ Marcio De Moraes, ${ }^{1}$ José Ricardo de Albergaria-Barbosa, ${ }^{1}$ Roger Willian Moreira ${ }^{1}$} sidad Estatal de Campinas, São Paulo, Brasil.

2.Facultad de Medicina de la Universidad de la Frontera, Temuco-Chile.

\section{Correspondencia:}

José Luis Muñante Cárdenas

Oral and Maxillofacial Surgery, Piracicaba Dental School, CP 52, State University of Campinas, 13414-903, Piracicaba, São Paulo, Brazil, Correo electrónico: jlmunante@fop.unicamp.br Fecha de recepción: 10-10-11

Fecha de aceptación:26-11-11

Fecha de recepción: 10-10-11

Fecha de aceptación:26-11-11

\section{Introducción}

Conducir una bicicleta puede proporcionar un cierto grado de independencia y de libertad, elementos que representan sin duda un cambio importante en la vida del niño. Es así como la bicicleta se convierte en un juego popular de recreación y transporte entre los niños y adolescentes. Paralelamente, se convierte también en la principal causa de accidentes graves tratados en los centros de emergencia.

En Norteamérica, cada año cerca de 1,000 personas mueren debido a accidentes ciclísticos y cerca de medio millón de personas son atendidas en los centros de emergencia a causa de estos accidentes. Alrededor de $75 \%$ de los pacientes sufren traumatismos o fracturas cráneo-maxilofaciales. ${ }^{2}$

Los índices de accidentes ciclísticos con traumatismo facial grave y muerte son de 3 a 6 veces más altos en nińos y adolescentes cuando comparado con otros grupos etarios. 1 Además, aproximadamente $65 \%$ de estos accidentes ocurren en adolescentes menores de 15 años. ${ }^{3}$

Diversos estudios demuestran que los accidentes ciclísticos representan la principal etiología del trauma facial, siendo su incidencia reducida significativamente cuando los ciclistas hacen uso de dispositivos de seguridad, como el casco protector. ${ }^{2,3,4}$

El objetivo de este estudio fue evaluar la incidencia, uso de dispositivo de seguridad, tipo y severidad de traumatismo maxilofacial, tratamiento y complicaciones registradas en pacientes víctimas de accidentes ciclísticos en una región del Estado de São Paulo. Los resultados obtenidos son importantes para impulsar programas orientados a la prevención de accidentes en este grupo etario.

\section{Material y método}

La muestra para el presente estudio fue seleccionada a partir del análisis de 2986 historias clínicas de pacientes víctimas de traumatismos faciales atendidos por el Área de Cirurgía Buco Máxilo Facial de la Facultad de Odontologia de Piracicaba, Universidad Estatal de Campinas de 1999 a 2008. Fueron selecionados todos los pacientes en edad pediátrica y adolescente (de 0 a 18 anos) que sufrieron trauma facial por accidentes ciclísticos.

Fueron analizadas las siguientes variables: género, grupo etario, uso de dispositivos de seguridad, tratamientos instituidos y complicaciones.

Los pacientes fueron agrupados en tres grupos etarios: Infantes e pré escolares: de 0 a 5 anos; edad escolar: de 6 a 12 anos; adolescentes: 13 a 18 anos. Los tratamientos instituidos fueron 
divididos en a) Conservador, o reducción cerrada; b) quirúrgico o reducción abierta.

Las fracturas maxilofaciales fueron divididas de acuerdo a la región afectada en: Fracturas de tercio medio y fracturas de mandíbula. Las fracturas de tercio medio fueron divididas en: fracturas de maxila, del complejo zigomáticomaxilar, del complejo naso-órbito-etmoidal (NOE) y fracturas de piso de órbita. Fracturas nasales aisladas o en asociación con otras estructuras faciales también fueron incluídas en este estudio. Las fracturas de mandíbula fueron divididas en: Fracturas de proceso coronoide, cóndilo, sínfisis, parasínfisis, ramo y ángulo mandibular.

Un sistema de registro de datos informatizado fue creado basado en software Microsoft Access. Las variables fueron sistematizadas y las nomenclaturas padronizadas. Después que todas las historias clínicas fueron incluídas en el sistema, los datos fueron exportados para el software Microsoft Excel y sometidos a cálculos estadísticos con el software Biostat 3.0.

\section{Resultados}

Edad y distribucion por sexo. Fueron identificados $220(29,6 \%)$ pacientes víctimas de accidentes ciclísticos. De éstos, 156 pacientes fueron del género masculino y 64 fueron del género femenino (2.4:1). El grupo de los adolescentes fue el mas afectado (120 pacientes, $54.5 \%$ ), seguido por el grupo escolar (82 pacientes, $37.3 \%$ ) y por el grupo de los infantes (18 pacientes, $8.2 \%$ ).

Uso de dispositivos de seguridad. Solo 4 pacientes (1.8\%) estaban usando dispositivos de seguridad al momento del accidente.

Sitio de fractura. Fueron identificados 69 casos $(31.3 \%)$ de fracturas maxilofaciales. La mandíbula fue el hueso facial mas afectado con 38 casos, (55.1 $\%)$. Entre las fracturas mandibulares fueron observadas 10 fracturas complejas de mandíbula y 15 fracturas aisladas de cóndilo mandibular. Las fracturas de tercio médio fueron identificadas en 17 pacientes $(24.6 \%)$, de las cuales, 16 fueron del complejo zigomático orbitario y 1 fractura fue de tipo Le Fort I. Además, fueron observadas fracturas nasales en 14 casos (20.3 \%). Existió un marcado predominio del grupo de los adolescentes.

Tratamiento y complicaciones. Las fracturas aisladas de nariz, fracturas mandibulares y de tercio medio facial que presentaron poco desplazamiento y que no afectaron la estética y función fueron tratadas, en la gran mayoria de los casos, de forma conservadora $(50$ pacientes, $72.5 \%$ ). Diecinueve procedimientos quirúrgicos fueron realizados $(27.5 \%)$, de los cuales 16 (84.2\%) correspondieron a reducción abierta y fijación interna rígida (FIR) de fracturas mandibulares: 3 fracturas de sínfisis y 9 fracturas de parasínfisis; además, 4 fracturas condilares aisladas en pacientes adolescentes también fueron abordadas quirúrgicamente. En relación a las fracturas del tercio medio, 3 pacientes (15.8 $\%)$ fueron tratados quirúrgicamente: 2 fracturas de complejo zigomático orbitario y una fractura tipo Le Fort I. Ningún caso de óbito fue reportado.

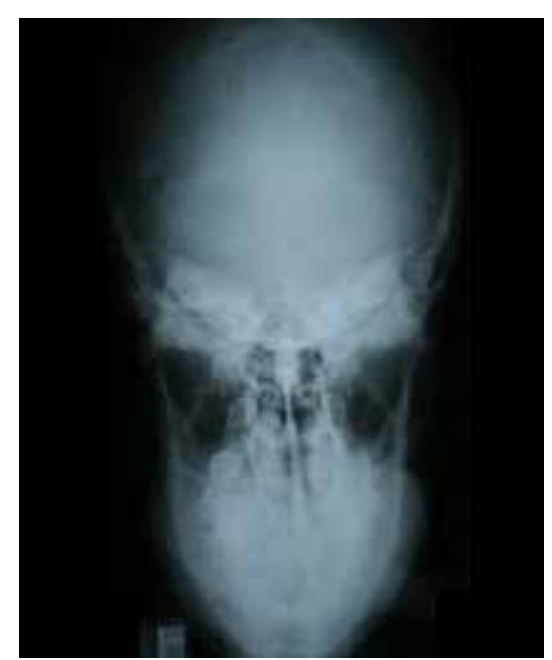

Figura 1. Radiografia de Towne, mostrando fractura de cóndilo mandibular derecho.

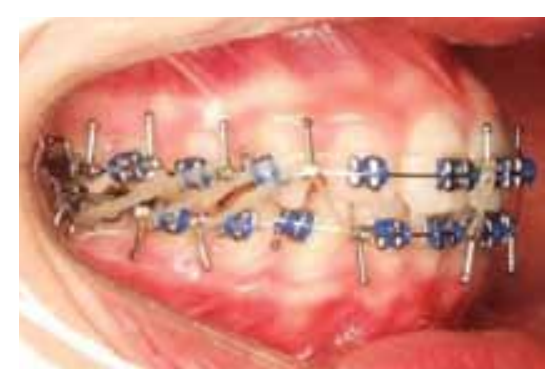

Figura 2. Tratamiento conservador o reducción cerrada de fractura condilar en el mismo paciente de la figura 1, mediante instalación de aparato ortodóntico y uso de elásticos para guiar la oclusión.

Complicaciones. Fue reportado un caso de parestesia del nervio alveolar inferior en un paciente adolescente del género masculino.

\section{Discusión}

Diversos estudios demostraron que la principal etiología de traumatismo en el territorio maxilofacial estaba relacionada a accidentes ciclísticos. ${ }^{5,6,7}$ Esta misma etiología fue encontrada en nuestra región de estudio, donde estos accidentes representaron $29 \%$ de los traumatismos faciales. Esto puede deberse a que las bicicletas representan un medio de transporte de uso extendido por los grupos etarios evaluados en el interior del Estado de São Paulo. De otro lado, y en concordancia con otros estudios 5,7 fue observado que los accidentes ciclísticos fueron predominantes en el grupo de los adolescentes.

En cuanto al género, estudios demuestran que el género masculino es más afectado que el femenino, manteniéndose constante este predominio en el tiempo. Las proporciones masculino/ femenino varían de 2:1 para $6: 15,7,8,9,10,11$. Nuestros resultados son consistentes con los reportados en la literatura, determinándose una relación masculino/ femenino de 2.4:1, siendo el género masculino predominante en todos los grupos etarios evaluados. Esto puede ser explicado debido a que los niños del género masculino están más relacionados a las actividades físicas de contacto y con actividades deportivas. ${ }^{10,12}$

En referencia a la edad, los infantes pasan mayor tiempo protegidos por el entorno familiar y por tanto menos expuesto a probables accidentes que podrían causar fracturas en otros grupos etarios. ${ }^{13}$ Pero, a medida que los niños crecen, su ambiente social cambia: van a la escuela, participan de deportes y actividades lúdicas de contacto, tienen cierta autonomía para poder trasladarse a otros lugares, conducen triciclos y bicicletas. Su altura, peso, fuerza y agresividad también aumentan, sus senos paranasales se desarrollan y su face se vuelve menos flexible y menos protegida por la prominencia frontal del cráneo, siendo más expuesta a fracturas. 14,15 Por tanto, la incidencia de traumatismos maxilofaciales se incrementa gradualmente con la edad 5,7,12,16,17,18 Este mismo comportamiento fue también observado en en los pacientes de este estudio, donde la incidencia de traumatismos fue de 18 pacientes en el grupo de los infantes, pasando a 82 en el grupo escolar y a 120 en el grupo de los adolescentes.

Según la literatura, las fracturas de mandíbula son las más comunes en la región maxilofacial en pacientes pediátricos y adolescentes. ${ }^{7,16,17,19,} 20$ Esta vulnerabilidad de la mandíbula probablemente se deba a que se trata de un hueso móvil y por tanto con menor soporte óseo cuando comparado con los huesos del tercio medio facial. ${ }^{12,21}$ Los datos obtenidos en este estudio también confirman este padrón, reportándose a la 
mandíbula como el hueso más afectado por fracturas faciales.

De otro lado, el tercio medio facial es el área más protegida en niños debido a su posición retraída en relación a un cráneo prominente que, juntamente a la mandíbula, provén protección y absorben la mayoría de los impactos traumáticos ${ }^{6,20}$. Debido a estos factores, las fracturas de tercio medio son aún más raras en niños, variando su incidencia de $0.5 \%$ a $25 \%$. ${ }^{8,20} \mathrm{Al}$ igual que en esos estudios, nosotros identificamos $24.6 \%$ de fracturas en esta región, siendo comúnmente afectado el hueso zigomático. Las fracturas tipo Le Fort fueron menos comunes y generalmente relacionadas al grupo de los adolescentes. Es interesante observar como una fractura tipo Le Fort I, relacionada generalmente a accidentes de tránsito de alta energía ${ }^{20}$, tenga como etiología al accidente ciclístico, lo que denota la gran violencia de estos traumatismos.

Cincuenta pacientes recibieron tratamiento conservador, representando el $72.4 \%$. Los resultados que se obtuvieron difieren de los reportados por Holland (2001) ${ }^{22}$, quien afirmó que la identificación de fracturas faciales en niños era importante debido a que más de la mitad de ellas iría a exigir algún tipo de reducción abierta. Los criterios para el uso del tratamiento conservador en nuestro Servicio fueron: poco o mínimo desplazamiento óseo, una reproducción adecuada de la oclusión y ausencia de déficit de función. Además, el alto índice de procedimientos prefiriendo la reducción cerrada está de acuerdo con los tratamientos actualmente propuestos por la literatura, presentando resultados satisfactorios a largo plazo.,23

Diversos estudios identificaron un alto grado de desplazamiento en las fracturas causadas por vehículos motorizados ${ }^{9,20}$. En este estudio en cambio, se verificó que un alto número de fracturas desplazadas tuvieron como etiología a los accidentes ciclísticos. Es así como, de las 69 fracturas identificadas, 19 casos (27 $\%)$ presentaron amplio desplazamiento de los fragmentos óseos, alteración de la oclusión y compromiso estético, condiciones que indicaron la reducción abierta y FIR de estas fracturas.

Las complicaciones resultantes de fracturas faciales en pacientes pediátricos son raras y, cuando se presentan, están relacionadas generalmente a casos de severa conminución o a fracturas con gran desplazamiento de los fragmentos óseos. ${ }^{21}$ En este estudio, el único caso de complicación registrado fue parestesia del nervio alveolar inferior. El paciente fue tratado mediante aplicaciones locales diarias de laser de baja intensidad durante 01 semana, mostrando evolución favorable.

La gravedad de las fracturas que necesitaron de reducción abierta podría ser explicada por la violencia de los impactos y por la falta de dispositivos de seguridad en la gran mayoría de nuestros pacientes. De hecho, al momento del accidente, solo el $1.8 \%$ de los afectados estaban usando algún tipo de dispositivo de seguridad. Estos datos son coincidentes con los reportados por Ferreira (2004) ${ }^{20}$ quien relató el incumplimiento de la legislación existente en temas de seguridad vial en los casos de accidentes ciclísticos registrados en una población portuguesa. Otro estudio reportó que solamente $3 \%$ de ciclistas adolescentes entre 14-15 años usaron algún tipo de dispositivo de seguridad. ${ }^{24}$

El uso de dispositivos de seguridad, como cascos protectores, previenen los traumatismos cranianos en cerca del 85 $\%$ y han demostrado ser efectivos en la reducción de traumatismos faciales se$\operatorname{veros}^{3,25}$. Desde que su uso en diversos países fue reglamentado por Ley, estos dispositivos han reducido el número de muertes o accidentes graves motivados por bicicleta en niños e infantes hasta en un $61 \%$ 26,27. Es así como en 1995 la Academia Americana de Pediatría recomendó el uso obligatorio de cascos protectores para los ciclistas. ${ }^{28}$

En Brasil, así como en muchos países latinoamericanos, el uso obligatorio de dispositivos de seguridad para ciclistas es aplicado parcialmente para actividades deportivas o profesionales. Nosotros consideramos que son necesarias políticas públicas más incisivas en relación a temas de educación y prevención de accidentes, así como una adecuada legislación para el cumplimiento de las normas ya existentes en materia de seguridad vial, sobre todo en cuanto al uso obligatorio de estos dispositivos para ciclistas en actividades recreativas o que usen bicicletas como medio de transporte urbano. Estas medidas pueden contribuir decisivamente en la reducción de la severidad de lesiones maxilofaciales y en la disminución de los costos de atención médica en el Sistema Público de Salud.

\section{Conclusiones}

Los accidentes ciclísticos representaron la principal etiología del trauma maxilofacial en la población de estudio. Este alto índice, así como la severidad de las lesiones, podria estar relacionado con la ausencia de dispositivos de seguridad en los ciclistas. El tratamiento conservador fue utilizado en la mayoría de los casos.

La prevención, mediante el uso dispositivos de seguridad, es una manera simple y efectiva de minimizar la gravedad de las lesiones, representando un costobeneficio bastante interesante para los servicios públicos de Salud, principalmente en países en vías de desarrollo.

Agradecimientos:

Los autores desean expresar su agradecimiento a:

Coordenação de Aperfeiçoamento de Pessoal de Nível Superior (CAPES) por el apoyo económico para realizar el presente estudio.

Prof. Dra. Erika Harth-Chú por su ayuda en el desarrollo y corrección del manuscrito.

\section{Referencias bibliográficas}

1. Shafi S, Gilbert JC, Loghmanee F, Allen JE, Caty MG, Glick PL, Carden S, Azizkhan RG. Impact of bicycle helmet safety legislation on children admitted to a regional pediatric trauma center. J Pediatr Surg. 1998; 33(2):317-21.

2. Sacks JJ, Holmgreen P, Smith SM, Sosin DM Bicycle-associated head injuries and deaths in the United States from 1984 through 1988. How many are preventable? JAMA. 1991;266(21):3016-3018

3. Thompson DC, Rivara FP, Thompson RS. Effectiveness of bicycle safety helmets in preventing head injuries. A case-control study. JAMA. 1996 25;276(24):1968-73

4. Berg P, Westerling R. Bicycle helmet use among schoolchldren--the influence of parental involvement and children's attitudes. Inj Prev. 2001;7(3):218-222.

5. Maniglia AJ, Kline SN. Maxillofacial trauma in the pediatric age group. Otolaryngol Clin North Am. 1983; 16(3):717-730.

6. Carroll MJ, Hill CM, Mason DA. Facial fractures in children. Br Dent J. 1987; 11(1): 23-26.

7. Bamjee Y, Lownie JF, Cleaton-Jones PE, Lownie MA. Maxillofacial injuries in a group of South Africans under 18 years of age. $\mathrm{Br} \mathrm{J}$ Oral 
Maxillofac Surg. 1996; 34(4):298302.

8. Kaban LB. Diagnosis and treatment of fractures of the facial bones in children 1943-1993.J Oral Maxillofac Surg. 1993; 51(7):722-729.

9. Hardt N.,Gottsauner A. The treatment of mandibular fractures in children.

J Craniomaxillofac Surg. 1993;21(5):214-219.

10. Oji C. Jaw fractures in Enugu, Nigeria, 1985-95. Br J Oral Maxillofac Surg. 1999;37(2):106-9.

11. Infante Cossio P, Espin Galvez F, Gutierrez Perez JL, Garcia-Perla A, Hernandez Guisado JM. Mandibular fractures in children. A retrospective study of 99 fractures in 59 patients. Int J Oral Maxillofac Surg. 1994; 23(6): 329-331.

12. Qudah MA, Al-Khateeb T, Bataineh AB, Rawashdeh MA. Mandibular fractures in Jordanians: a comparative study between young and adult patients.J Craniomaxillofac Surg. 2005; 33(2): 103-106.

13. Thorén H, Iso-Kungas P, Iizuka T, Lindqvist C, Törnwall J . Changing trends in causes and patterns of facial fractures in children. Oral Surg Oral Med Oral Pathol Oral Radiol Endod. 2009; 107(3):318-324.

14. Zimmermann CE, Troulis MJ, Kaban LB. Pediatric facial fractures: recent advances in prevention, diagnosis and management. Int J Oral Maxillofac Surg. 2005; 34(8): 823833.
15. Zerfowski M, Bremerich A. Facial trauma in children and adolescents. Clin Oral Investig. 1998; 2(3): 120-124.

16. Zachariades N, Mezitis M, Mourouzis C, Papadakis D, Spanou A.Fractures of the mandibular condyle: a review of 466 cases. Literature review, reflections on treatment and proposals. J Craniomaxillofac Surg. 2006; 34(7): 421-432.

17. Lida S, Matsuya T. Paediatric maxillofacial fractures: their aetiological characters and fracture patterns.J Craniomaxillofac Surg. 2002; 30(4):237-241.

18. Thorén $\mathrm{H}$, Iso-Kungas $\mathrm{P}$, Iizuka $\mathrm{T}$, Lindqvist C, Törnwall J . Changing trends in causes and patterns of $\mathrm{fa}$ cial fractures in children. Oral Surg Oral Med Oral Pathol Oral Radiol Endod. 2009; 107(3):318-324.

19. Posnick JC, Wells M, Pron GE. Pediatric facial fractures: evolving patterns of treatment. J Oral Maxillofac Surg. 1993; 51(8):836-844.

20. Ferreira P, Marques M, Pinho C, Rodrigues J, Reis J, Amarante J. Midfacial fractures in children and adolescents: a review of 492 cases. Br J Oral Maxillofac Surg. 2004;42(6):501-505.

21. Thorén $H$, Iizuka $T$, Hallikainen D, Lindqvist C. Different patterns of mandibular fractures in children. An analysis of 220 fractures in 157 patients. J Craniomaxillofac Surg. 1992; 20(7):292-296.

22. Holland AJ, Broome C, Steinberg A, Cass DT. Facial fractures in children Pediatr Emerg Care. 2001;17(3):157-160.

23. Eggensperger Wymann NM, Hölzle A, Zachariou Z, Iizuka T.J Pediatric craniofacial trauma. Oral Maxillofac Surg. 2008; 66(1): 5864.

24. Berg P, Westerling R. A decrease in both mild and severe bicycle-related head injuries in helmet wearing ages-trend analyses in Sweden. Health Promot Int. 2007;22(3):191-197.

25. Thompson DC, Nunn ME, Thompson RS, Rivara FP Effectiveness of bicycle safety helmets in preventing serious facial injury. JAMA. 1996 25;276(24):1974-1975.

26. Rodgers GB. Effects of state helmet laws on bicycle helmet use in children and adolescents. Inj Prev 2002;8:42-46.

27. Cameron MH, Vulcan AP, Finch $\mathrm{CF}$, et al: Mandatory bicycle helmet use following a decade of helmet promotion in Victoria, AustraliaAn evaluation. Accid Anal Prev 26:325-337, 1994

28. American Academy of Pediatrics, Committee on Injury and Poisoning Prevention: Bicycle helmets. Pediatrics 95:609-610, 1995

29. Tanaka N, Uchide N, Suzuki K, Tashiro T, Tomitsuka K, Kimijima Y, Amagasa TJ. Maxillofacial fractures in children. Craniomaxillofac Surg. 1993; 21(7):289-293. 